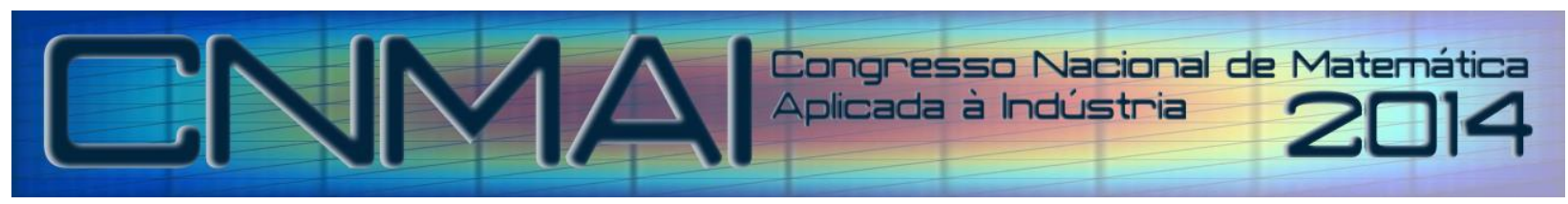

18 a 21 de novembro de 2014, Caldas Novas - Goiás

\title{
FATIGUE ANALYSIS OF STRUCTURES WITH PASSIVE CONSTRAINING DAMPING LAYERS SUBJECTED TO RANDOM LOADS
}

\author{
Ana Paula Rodrigues Ramos, anapaula@icte.uftm.edu.br ${ }^{1}$ \\ Antônio Marcos Gonçalves de Lima, amglima @ mecanica.ufu.br ${ }^{1}$ \\ ${ }^{1}$ Federal University of Uberlândia - School of Mechanical Engineering Campus Santa Mônica - P.O. Box 593, CEP \\ 38400-902 - Uberlândia/MG-Brazil
}

\begin{abstract}
Resumo: This paper is dedicated to the investigation of the possibility of increasing fatigue life of engineering structures subjected to multiaxial random loads by applying constrained viscoelastic layers. The rationale for such study is the fact that as the addition of viscoelastic materials provide decreased vibration amplitudes, it becomes important to quantify the increase of reliability that can be obtained. Moreover, despite the fact that many multiaxial fatigue damage criteria applicable to undamped structures exist in the literature, none of them is adapted to deal with the problem of estimation the fatigue damage in structures incorporating viscoelastic damping, since they must conveniently account for the frequency-and temperature-dependent behavior of the viscoelastic material. Due to the nature of the stress state of the considered problem, the fatigue damage is assessed by using Sine's global criterion. After presenting the theoretical aspects, the numerical fatigue damage analyses of a three-layer sandwich plate treated by passive constrained damping layer are addressed, and the main features of the methodology are discussed.
\end{abstract}

Palavras-chave: Viscoelastic damping, Sine's criterion, fatigue damage, passive control.

\section{INTRODUCTION}

In the context of new developments in many mechanical engineering domains, it is observed an increasing demand for durability, reliability, safety and comfort. As a result, during the design phase or during the analysis of an existing system, tests should be performed in order to predict its fatigue life over a desired range of loading conditions, since there is a high possibility to excite the resonance frequencies, which can lead to fatigue failure. As an example, longspan bridges that have performed well under wind loads for decades have been recently found to have accumulated fatigue damage resulting in cracking of the structural components (Palmeri and Ricciardelli, 2006).

More recently, much effort has been devoted to the study of efficient passive control techniques to be applied in medium- to high-scale structures to mitigate their undesired levels of vibration and extending their fatigue life. Among these, passive control techniques based on the use of viscoelastic materials present some advantages such as inherent stability, effectiveness in broad frequency bands and moderate development and maintenance costs (Nashif and Jones, 1985; Rao, 2001; Samali and Kwok, 1995). In practice, these materials can be applied either as discrete devices, such as translational mounts and rotational joints, or surface treatments known as Passive Constraining Layer Damping (PCLD), and Active Constraining Layer Damping (ACLD) (Nashif and Jones, 1985; Christensen, 1982). However, the incorporation of the viscoelastic behavior into the finite element (FE) models and the numerical resolution of the resulting equations of motion are particularly relevant aspects of the modeling procedures since for viscoelastic structures the stiffness matrix is frequency- and temperature-dependent. Besides the standard rheological and more complex models (Nashif and Jones, 1985) intended to represent the dynamic behavior of viscoelastic materials, the complex modulus approach has been adopted in this paper, since it is adequate for frequency responses analyses which are considered to be relevant parameters when the fatigue life of structural components are to be estimated.

Several multiaxial fatigue criteria have been developed for performing fatigue damage analysis of elastic structures, as reported in references (You and Lee, 1996; Pitoiset and Preumont, 2000; Lambert et al., 2010). However, applications to the case of structures subjected to multiaxial random stresses in the frequency domain are not numerous (Pitoiset and Preumont, 2000; Dan Vang, 1993, Lambert et al., 2010). Moreover, none of them appears to be completely satisfactory to deal with the problem of estimation the fatigue life of viscoelastic structures, which motivate the study reported in this paper. In particular, Pitoiset and Preumont (2000) have contributed successfully with the formulas of the Crossland and Matake criteria in the frequency domain. However, the proposed approach have some limitations such as being confined to zero mean random loads and narrow band stress responses. Lambert et al. (2010), based on the fact that the Sines's global criterion gives better correlations with the results obtained by experimental tests (Weber, 1999), 
have proposed a probabilistic approach of the Sines' criterion by considering the effect of the load phase shifts on fatigue damage. This probabilistic model is employed in this paper in order to characterize the statistical distributions of Sines' damage indicator of viscoelastic structures subjected to random excitations.

The typically high dimensions of large-scale FE models of industrial structures incorporating viscoelastic materials make the numerical damage analysis of such systems very costly, sometimes unfeasible. This fact motivates the use of a special condensation method based on the use of a constant (frequency- and temperature-independent) reduction basis which takes into account a priori information of the viscoelastic damping forces (de Lima et al., 2010).

In the remainder, after the presentation of the theoretical foundations, the description of a numerical investigation of the fatigue damage distribution of a rectangular plate treated by passive constrained layer damping subjected to a random transverse load is addressed.

\section{REVIEW OF THE MODELING OF PASSIVE CONSTRAINING LAYER DAMPING.}

In this section, the model of a moderately thin three-layer sandwich plate FE, which can be frequently found, for example, in aerospace systems, is summarized, based on the original developments made by Khatua and Cheung (1973) and implemented by de Lima et al. (2010). Fig. 1 depicts a rectangular element formed by an elastic base-plate (1), a viscoelastic core (2) and an elastic constraining layer (3). This element contains four nodes and seven DOFs per node, representing the in-plane displacements in the middle plane of the base-plate in directions $x$ and $y$ (denoted by $u_{1}$ and $v_{1}$, respectively), the in-plane displacements of the middle plane of the constraining layer in directions $x$ and $y$ (denoted by $u_{3}$ and $v_{3}$, respectively), the transverse displacements, $w$, and the cross-section rotations about $x$ and $y$, denoted by $\theta_{x}$ and $\theta_{y}$, respectively.

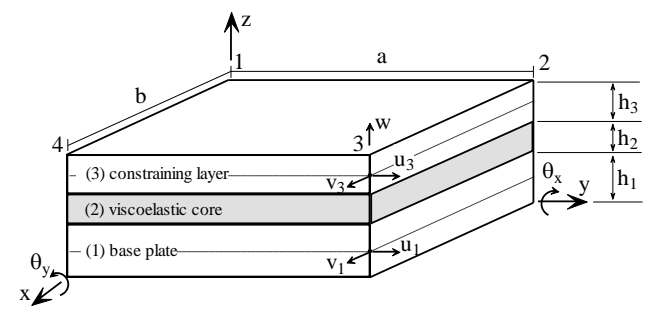

Figure 1: Illustration of the three-layer sandwich plate element.

In the development of the theory, the following assumptions are adopted: (i) all the materials involved are homogeneous and isotropic and present linear mechanical behavior; (ii) normal stresses and strains in direction $z$ are neglected for all the three layers; (iii) the elastic layers (1) and (3) are modeled according to Kirchhoff's theory; (iv) for the viscoelastic core, Mindlin's theory is adopted (transverse shear is included); (v) the cross-section rotations $\theta_{x}$ and $\theta_{y}$ are assumed to be the same for the elastic layers; $(v i)$ the transverse displacement $w$ is the same for all the three layers. These assumptions have been considered by many authors as being adequate for the modeling of thin panels, as it is the case of the structures addressed in the present paper, where it is assumed the facesheets to be thin. Moreover, previous studies carried-out by the authors have demonstrated good correlation between model predictions and their experimental counterparts.

The discretization of the displacement fields within the element is made by using linear interpolation functions for the displacements in the middle plane of the elastic layers in directions $x$ and $y$, and a cubic interpolation function for the transverse displacement, according to the general relation $\boldsymbol{u}(x, y, t)=\boldsymbol{N}(x, y) \boldsymbol{u}_{(e)}(t)$, where $\boldsymbol{N}(x, y)$ is the matrix formed by the shape interpolation functions, and $\boldsymbol{u}_{(e)}(t)=\left[u_{1}^{i} v_{1}^{i} u_{3}^{i} v_{3}^{i} w^{i} \theta_{x}^{i} \theta_{y}^{i}\right]^{T}$ with $i=1$ to 4 represents the vector containing the mechanical nodal variables as a function of time. The strain-displacement relations, $\boldsymbol{\varepsilon}(x, y, z, t)=\boldsymbol{B}(x, y, z) \boldsymbol{u}_{(e)}(t)$, are used and the resulting strains for elastic layers $(k=1,3) \varepsilon^{(k)}=\left[\begin{array}{lll}\varepsilon_{x}^{(k)} & \varepsilon_{y}^{(k)} & \gamma_{x y}^{(k)}\end{array}\right]^{T}$ and for the viscoelastic core $\varepsilon^{(2)}=\left[\begin{array}{lllll}\varepsilon_{x}^{(2)} & \varepsilon_{y}^{(2)} & \gamma_{x y}^{(2)} & \gamma_{x z}^{(2)} & \gamma_{y z}^{(2)}\end{array}\right]^{T}$ are generated. Thus, based on the stress-states assumed for each layer and the stress-strain relations, the stress response of the system can be obtained as:

$$
\boldsymbol{s}(x, y, z, t)=\boldsymbol{C}^{*} \boldsymbol{\varepsilon}(x, y, z, t)=\boldsymbol{C}^{*} \boldsymbol{B}(x, y, z) \boldsymbol{u}_{(e)}(t)
$$

where for elastic layers $\boldsymbol{C}^{*}=\boldsymbol{C}$ is the isotropic elastic material properties matrix, and for the viscoelastic core, $\boldsymbol{C}^{*}=\boldsymbol{C}(\omega, T)$ represents the frequency- and temperature-dependent material properties. Matrix $\boldsymbol{B}(x, y, z)$ is formed by differential operators appearing in the strain-displacement relations, as detailed in reference (de Lima et al., 2010). 
The strain and kinetic energies of the three-layer sandwich plate FE can be formulated and the elementary mass and stiffnesses matrices are generated as follow:

$$
\begin{gathered}
\boldsymbol{M}^{(e)}=\sum_{k=1}^{3} \rho^{(k)} h^{(k)} \int_{x=0}^{a} \int_{y=0}^{b} \boldsymbol{N}^{T}(x, y) \boldsymbol{N}(x, y) d y d x \\
\boldsymbol{K}_{e}^{(e)}=\sum_{k=1,3} \int_{z=0}^{h^{(k)}} \int_{x=0}^{a} \int_{y=0}^{b} \boldsymbol{B}^{T}(x, y, z) \boldsymbol{C} \boldsymbol{B}(x, y, z) d y d x d z \\
\boldsymbol{K}_{v}^{(e)}(\omega, T)=\int_{z=0}^{h^{(k)}} \int_{x=0}^{a} \int_{y=0}^{b} \boldsymbol{B}^{T}(x, y, z) \boldsymbol{C}(\omega, T) \boldsymbol{B}(x, y, z) d y d x d z
\end{gathered}
$$

where $h^{(k)}$ and $\rho^{(k)}$ are the thickness and the mass density of the $k$-th layer, respectively. The stiffnesses matrices, $\boldsymbol{K}_{e}^{(e)}=\boldsymbol{K}_{1}^{(e)}+\boldsymbol{K}_{3}^{(e)}$ and $\boldsymbol{K}_{v}^{(e)}(\omega, T)=\boldsymbol{K}_{2}^{(e)}(\omega, T)$ are, respectively, the contributions of the purely elastic and viscoelastic parts of the structure.

From the elementary matrices computed for each element of the FE mesh, and neglecting other forms of damping, the global equations of motion are constructed, accounting for the node connectivity, using standard FE assembling procedures. After assembling, the global equations of motion in the frequency domain of the viscoelastic structure containing $N$ degrees-of-freedom (DOFs) can be expressed as follows:

$$
\left[\boldsymbol{K}_{e}+\boldsymbol{K}_{v}(\omega, T)-\omega^{2} \boldsymbol{M}\right] \boldsymbol{U}(\omega, T)=\boldsymbol{F}(\omega)
$$

where $\boldsymbol{M} \in R^{N \times N}$ is the mass (symmetric, positive-definite) matrix, and $\boldsymbol{K}_{e} \in R^{N \times N}$ and $\boldsymbol{K}_{v}(\omega, T) \in R^{N \times N}$ are the stiffness matrices (symmetric, nonnegative-definite) corresponding to the purely elastic and viscoelastic substructures, respectively. $\boldsymbol{U}(\omega, T) \in R^{N}$ and $\boldsymbol{F}(\omega) \in R^{N}$ are, respectively, the vectors of displacements and external loads.

At this point, the inclusion of the frequency-dependent behavior of the viscoelastic material can be made by using the so-called Elastic-Viscoelastic Correspondence Principle (Christensen, 1982), according to which, for a given temperature, matrix $\boldsymbol{K}_{v}(\omega, T)$ can be first generated for specific types of finite elements (rods, beams, plates, etc.) assuming that the longitudinal modulus and/or the shear modulus (according to the stress-state) are constant (independent on frequency and temperature). Then, after the finite element matrices are constructed, the frequencytemperature dependency of those moduli is introduced according to the complex modulus approach combined with the Frequency-Temperature Superposition Principle (Nashif and Jones, 1985). By assuming the widely accepted hypothesis of a constant (frequency-independent) Poisson ratio for the viscoelastic material, the longitudinal modulus $E(\omega, T)$ becomes proportional to the shear modulus $G(\omega, T)$ through the relation $G(\omega, T)=E(\omega, T) / 2(1+v)$. Then, one of the two moduli can be factored-out of the stiffness matrix of the viscoelastic sub-structure, according to $\boldsymbol{K}_{v}(\omega, T)=G(\omega, T) \overline{\boldsymbol{K}}_{v}$ (were $\overline{\boldsymbol{K}}_{v}$ is a frequency-independent matrix), which is combined with Eq. (3) to produce the complex Frequency Response Functions (FRFs) matrix:

$$
\boldsymbol{H}(\omega, T)=\left[\boldsymbol{K}_{\boldsymbol{e}}+G(\omega, T) \overline{\boldsymbol{K}}_{v}-\omega^{2} \boldsymbol{M}\right]^{-1}
$$

Thus, the time-domain stress response of the viscoelastic system can be computed according to (1) by taking into account the following relation:

$$
\boldsymbol{u}(t, T)=\int_{-\infty}^{+\infty} \boldsymbol{h}(\tau, T) \boldsymbol{f}(t-\tau) \mathrm{d} \tau
$$

where $\boldsymbol{h}(t, T)=\frac{1}{2 \pi} \int_{-\infty}^{+\infty} \boldsymbol{H}(\omega, T) e^{j \omega t} \mathrm{~d} \omega$ is the impulse response of the viscoelastic system.

\section{VISCOELASTIC CONDENSATION PROCEDURE}

In the case of FE models of complex structures of industrial interest constituted by a large number of DOFs (hundreds of thousand or even millions), it becomes practically impossible to compute the FRFs directly from Eq. (4), owing to the prohibitive computation times and storage memory required. This fact motivates the use of model reduction procedures, which aim at reducing the model dimensions and the associated computational burden, while keeping a reasonable predictive capacity of the numerical models. This can be done based on the assumption that the exact responses, given by the resolution of Eq. (3), can be approached by projecting the response vector on a reduced basis as follows: 


$$
\boldsymbol{U}(\omega, T)=\boldsymbol{T} \hat{\boldsymbol{U}}(\omega, T)
$$

where $\boldsymbol{T} \in C^{N \times N R}$ is the transformation matrix formed column-wise by a vector basis, $\hat{\boldsymbol{U}}(\omega, T) \in C^{N R}$ are generalized coordinates, and $N R<<N$ is the number of reduced vectors in the basis. Thus, the reduced FRF and stress response matrices can be written as follow:

$$
\hat{\boldsymbol{H}}(\omega, T)=\left[\hat{\boldsymbol{K}}_{e}+G(\omega, T) \hat{\boldsymbol{K}}_{v}-\omega^{2} \hat{\boldsymbol{M}}\right]^{-1}
$$

where $\hat{\boldsymbol{K}}_{e}=\boldsymbol{T}^{T} \boldsymbol{K}_{e} \boldsymbol{T}, \hat{\overline{\boldsymbol{K}}}_{v}=\boldsymbol{T}^{T} \overline{\boldsymbol{K}}_{v} \boldsymbol{T}$ and $\hat{\boldsymbol{M}}=\boldsymbol{T}^{T} \boldsymbol{M T} . \boldsymbol{\Psi}=\boldsymbol{C}^{*} \boldsymbol{B} \boldsymbol{T}$ is the reduced stress matrix.

For systems containing viscoelastic materials, the selection of the reduction basis is delicate as, owing to the dependence of the stiffness matrix with respect to frequency, the reduction basis should be able to represent the changes of the dynamic behavior as frequency is varied in the band of interest (de Lima et al., 2010). In this work, the strategy consists in using a reduction basis formed by a constant modal basis of the associate conservative system enriched by static residual vectors to account for the effects of the external loads and the viscoelastic damping forces. These static responses are computed from the low-frequency asymptotic stiffness matrix, representing the static behavior of the viscoelastic materials, which is given by:

$$
\boldsymbol{K}_{0}=\boldsymbol{K}_{e}+G_{0} \overline{\boldsymbol{K}}_{v}
$$

where $G_{0}$ is the real part of the modulus function obtained by the extrapolation of the modulus curves in the low frequency range by asymptotes, as shown in Fig. 2.

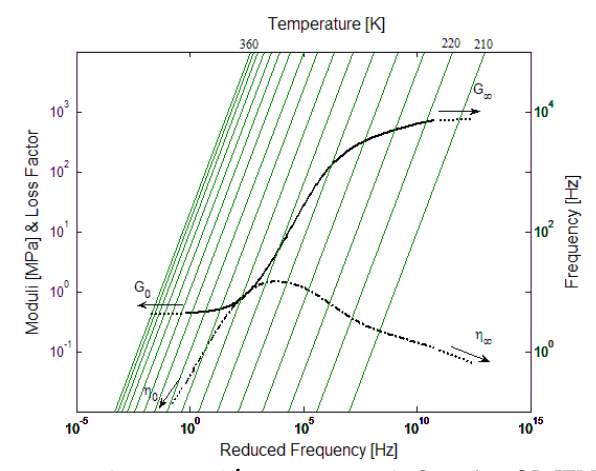

Figure 2: Master curves $\left(\longrightarrow G^{\prime} ; \boldsymbol{-} \cdot \boldsymbol{\eta}\right)$ ) for the 3MTM ISD112 material.

Then, the nominal basis can be obtained by the resolution of the eigenvalue problem:

$$
\begin{aligned}
& \left(\boldsymbol{K}_{0}-\lambda_{i} \boldsymbol{M}\right) \varphi_{i}=0 \quad i=1, \ldots, N \\
& \boldsymbol{\varphi}_{0}=\left[\begin{array}{llll}
\varphi_{1} & \varphi_{2} & \ldots & \varphi_{N R}
\end{array}\right], \quad \Lambda_{0}=\operatorname{diag}\left(\lambda_{1}, \ldots, \lambda_{N R}\right)
\end{aligned}
$$

It has been shown in previous studies (de Lima et al., 2010) that the number of necessary projection vectors can be reduced by introducing the residues associated to the viscoelastic damping forces, $\boldsymbol{R}_{v}^{0}=\boldsymbol{K}_{0}{ }^{-1} \overline{\boldsymbol{K}}_{v} \boldsymbol{\varphi}_{0}$, and the residues formed by the static displacements due to external forces, $\boldsymbol{R}=\boldsymbol{K}_{0}{ }^{-1} \boldsymbol{b}$, where $\boldsymbol{b} \in R^{N \times f}$ is a Boolean matrix which enables to select, among the DOFs, those in which the excitation forces are applied. These residuals are interpreted as the columns of the flexibility matrix of the associated undamped system, associated to the coordinates of application of two types of forces to it: the external excitation forces and the damping forces.

Thus, the enriched basis of reduction is given as follows:

$$
\boldsymbol{T}=\left[\begin{array}{lll}
\varphi_{0} & \boldsymbol{R} & \boldsymbol{R}_{v}^{0}
\end{array}\right]
$$

\section{STRESS RESPONSE OF THE VISCOELASTIC SYSTEM SUBJECTED TO A RANDOM LOAD}

\subsection{Mean value of the stress response.}


By combining equations (1) and (5), the mean value of the stress response of the viscoelastic structure subjected to a stationary random load, $f(t)$, is given by:

$$
\overline{\boldsymbol{s}}(t, T)=E\left[\int_{-\infty}^{+\infty} \boldsymbol{C}^{*} \boldsymbol{B h}(\tau, T) \boldsymbol{f}(t-\tau) \mathrm{d} \tau\right]=\boldsymbol{C}^{*} \boldsymbol{B}\left[\int_{-\infty}^{+\infty} \boldsymbol{h}(\tau, T) \mathrm{d} \tau\right] \overline{\boldsymbol{f}}(t)
$$

where $\overline{\boldsymbol{s}}(t, T)=E[s(t, T)]$ and $\overline{\boldsymbol{f}}(t)=E[\boldsymbol{f}(t)] \mathrm{E}[\bullet]$ is the mathematical expectation operator.

By applying the Fourier Transform for $\omega_{0}=0$, Eq. (11) assumes the following form:

$$
\overline{\boldsymbol{s}}(t, T)=\boldsymbol{C}^{*} \boldsymbol{B} \boldsymbol{H}\left(\omega_{0}, T\right) \overline{\boldsymbol{f}}(t)=\boldsymbol{C}^{*} \boldsymbol{B} \boldsymbol{K}_{0}^{-1} \overline{\boldsymbol{f}}(t)
$$

Equation (12) shows that the mean value of the stress response of a viscoelastic system subjected to stationary random loads are proportional to the mean value of the random loads and to the static response of the viscoelastic system computed by using the tangent stiffness matrix representing the static behavior of the viscoelastic materials, as can be seen in Fig. 2.

\subsection{Power spectral density matrix of the stress response.}

For the characterization of stationary random process the statistical quantity normally employed is the so-named power spectral density (PSD). For the viscoelastic system, the PSD matrices associated to the random load and response, $\boldsymbol{\Phi}_{f}(\omega) \in R^{N \times N}$ and $\boldsymbol{\Phi}_{u}(\omega, T) \in R^{N \times N}$, can be calculated, respectively, as follows (Lambert et al., 2010):

$$
\boldsymbol{\Phi}_{f}(\omega)=\int_{-\infty}^{+\infty} \boldsymbol{R}_{f}(\tau) \mathrm{e}^{-j \omega \tau} \mathrm{d} \tau, \quad \boldsymbol{\Phi}_{u}(\omega, T)=\int_{-\infty}^{+\infty} \boldsymbol{R}_{u}(\tau, T) \mathrm{e}^{-j \omega \tau} \mathrm{d} \tau
$$

where $\boldsymbol{R}_{f}(\tau)=\mathrm{E}\left[\boldsymbol{f}(t) \boldsymbol{f}(t+\tau)^{\mathrm{T}}\right] \in R^{N \times N}$ and $\boldsymbol{R}_{u}(\tau, T)=\mathrm{E}\left[\boldsymbol{u}(t, T) \boldsymbol{u}(t+\tau, T)^{\mathrm{T}}\right] \in R^{N \times N}$ are the correlation function matrices associated to the random forces and the random responses, respectively. Taking into account the relation (5), the correlation function matrix of the random response is given as:

or,

$$
\boldsymbol{R}_{u}(\tau, T)=E\left[\int_{-\infty-\infty}^{+\infty} \int_{-\infty} \boldsymbol{h}(\lambda, T) \boldsymbol{f}(t-\lambda) \boldsymbol{f}(t+\tau-\zeta)^{T} \boldsymbol{h}(\zeta, T)^{T} \mathrm{~d} \lambda \mathrm{d} \zeta\right]
$$

$$
\boldsymbol{R}_{u}(\tau, T)=\int_{-\infty}^{+\infty} \int_{-\infty}^{+\infty} \boldsymbol{h}(\lambda, T) \boldsymbol{R}_{f}(\tau+\lambda-\zeta) \boldsymbol{h}(\zeta, T)^{T} \mathrm{~d} \lambda \mathrm{d} \zeta
$$

where $\int_{-\infty}^{+\infty} \boldsymbol{f}(t-\lambda) \boldsymbol{f}(t+\tau-\zeta)^{T} \mathrm{dt}=\int_{-\infty}^{+\infty} \boldsymbol{f}(t) \boldsymbol{f}(t+\tau+\lambda-\zeta)^{T} \mathrm{dt}=\boldsymbol{R}_{f}(\tau+\lambda-\zeta)$ is valid for stationary random process.

Upon introduction of the Eq. (15) into relation (13), and considering the Inverse Fourier Transform of the PSD of the random loads, $\boldsymbol{R}_{f}(\tau+\lambda-\zeta)=\frac{1}{2 \pi} \int_{-\infty}^{+\infty} \boldsymbol{\Phi}_{f}(\omega) e^{i \omega(\tau+\lambda-\zeta)} d \omega$, it is possible to show that the PSD matrix of the random response can be obtained as follows:

$$
\boldsymbol{\Phi}_{u}(\omega, T)=\int_{-\infty}^{+\infty}\left\{\int_{-\infty}^{+\infty} \int_{-\infty}^{+\infty} \boldsymbol{h}(\tau, T)\left[\frac{1}{2 \pi} \int_{-\infty}^{+\infty} \boldsymbol{\Phi}_{f}(\omega) e^{i \omega(\tau+\lambda-\zeta)} d \omega\right] \boldsymbol{h}(\zeta, T)^{T} \mathrm{~d} \lambda \mathrm{d} \zeta\right\} e^{i \omega \tau} d \tau
$$

which can be reduced, after some mathematical manipulations, to the following expression:

$$
\boldsymbol{\Phi}_{u}(\omega, T)=\boldsymbol{H}(\omega, T) \boldsymbol{\Phi}_{f}(\omega) \boldsymbol{H}(\omega, T)^{T}
$$

Hence, taking into account Eq. (7), the reduced PSD matrix of the stress response can be formulated as:

$$
\hat{\boldsymbol{\Phi}}_{s}(\omega, T)=\boldsymbol{\Psi} \hat{\boldsymbol{H}}(\omega, T) \hat{\boldsymbol{\Phi}}_{f}(\omega) \hat{\boldsymbol{H}}(\omega, T)^{T} \boldsymbol{\Psi}^{T}
$$


where $\hat{\boldsymbol{\Phi}}_{f}(\omega)=\boldsymbol{T}^{T} \boldsymbol{\Phi}_{f}(\omega) \boldsymbol{T}$ and $\hat{\boldsymbol{\Phi}}_{s}(\omega, T)=\left[\begin{array}{ccc}\hat{\boldsymbol{\Phi}}_{x x, x x}(\omega, T) & \hat{\boldsymbol{\Phi}}_{x x, y y}(\omega, T) & \hat{\boldsymbol{\Phi}}_{x x, x y}(\omega, T) \\ & \hat{\boldsymbol{\Phi}}_{y y, y y}(\omega, T) & \hat{\boldsymbol{\Phi}}_{y y, x y}(\omega, T) \\ s y m & & \hat{\boldsymbol{\Phi}}_{x y, x y}(\omega, T)\end{array}\right]$.

\section{FATIGUE DAMAGE ESTIMATION OF A VISCOELASTIC SYSTEM}

In this section the Sines' global criterion initially developed for the fatigue damage analysis of elastic structures subjected to multiaxial loads is extended to the case of fatigue damage analysis of structures incorporating viscoelastic materials subjected to multiaxial random stress states. This can be done based on the statistical characteristics of the previously computed stress response of the viscoelastic system in the frequency domain.

The Sines' global criterion is expressed as follows (Sines, 1959):

$$
D_{\text {Sines }}=\frac{\sqrt{\boldsymbol{J}_{2}(t)}+\alpha \overline{\boldsymbol{p}}_{h}(t, T)}{\beta}
$$

where $\overline{\boldsymbol{p}}_{h}(t, T)=E\left[\boldsymbol{p}_{h}(t, T)\right]$ is the mean value of the hydrostatic pressure, $\sqrt{\boldsymbol{J}_{2}(t)}$ is the square root of the second invariant of the deviatoric stress tensor, and $\beta=t_{-1}$ and $\alpha=\frac{3 t_{-1}\left(R_{m}+f_{-1}\right)}{f_{-1} R_{m}}-\sqrt{6}$ are the parameters associated to the material. $f_{-1}$ and $t_{-1}$ represent, respectively, the endurance limits for the fully repeated stresses in tension and torsion for a number of cycles $N_{c}$, and $R_{m}$ is the failure strength of the material.

In Eq. (19), the effects of the mean stresses are considered through the computation of the mean value of the hydrostatic pressure. The mathematical expectation of the stress response $s(t, T)$ and the hydrostatic pressure of the system can be estimated, respectively, as follow:

$$
\begin{gathered}
E[\boldsymbol{s}(t, T)]=\overline{\boldsymbol{s}}(t, T)=\left[\bar{s}_{x x} \bar{s}_{y y} \bar{s}_{z z} \bar{s}_{x y} \bar{s}_{y z} \bar{s}_{x z}\right]^{T} \\
E\left[\boldsymbol{p}_{h}(t, T)\right]=\overline{\boldsymbol{p}}_{h}(t, T)=E\left[\frac{s_{x x}(t, T)+s_{y y}(t, T)+s_{z z}(t, T)}{3}\right]=\frac{1}{3}\left(\bar{s}_{x x}+\bar{s}_{y y}+\bar{s}_{z z}\right)
\end{gathered}
$$

In various fatigue analyses of systems subjected to proportional (in-phase) loads, the computation $\sqrt{\boldsymbol{J}_{2}(t)}=\|\boldsymbol{s}(t)\|$ is given by Euclidean norm of vector $s(t)=\left[s_{1}(t) s_{2}(t) s_{3}(t) s_{4}(t) s_{5}(t)\right]^{T}$, where $s_{1}(t)=\frac{\sqrt{3}}{2}\left[s_{x x}(t)-\frac{1}{3} s_{y y}(t)-\frac{1}{3} s_{z z}(t)\right]$, $s_{2}(t)=\frac{1}{2}\left[s_{y y}(t)-s_{z z}(t)\right], s_{3}(t)=s_{x y}(t), s_{4}(t)=s_{x z}(t)$ and $s_{5}(t)=s_{y z}(t)$. However, in the case of non-proportional (outof-phase) random loads, the fatigue analysis must take into account the random nature of the stress state and the fact that the direction and the length of vector $\boldsymbol{s}(t)$ are not constant over a load cycle. Thus, the computation $\sqrt{\boldsymbol{J}_{2}(t)}=\|\boldsymbol{s}(t)\|$ is no more applicable, and the following probabilistic approach of the Sines' criterion is retained (Lambert et al., 2010):

$$
E\left[D_{\text {Sines }}\right]=\frac{E\left[\sqrt{J_{2}(t, T)}\right]+\alpha \overline{\boldsymbol{p}}_{h}(t, T)}{\beta}
$$

In order to compute $E\left[\sqrt{J_{2}(t)}\right]$, the procedure adopted here is based on the strategy proposed by Bin Li and de Freitas (2002), where for a two-dimensional problem, it is considered a smaller ellipse in order to compute $\sqrt{J_{2}(t, T)}=\sqrt{R_{1}^{2}+R_{2}^{2}}$, where $R_{1}$ and $R_{2}$ are the semi-axes of the ellipse, as illustrated in Fig. 3.

In this paper, this procedure is generalized by considering an ellipsoid $\xi_{5}$ of five dimensions with the semi-axes $R_{i}(i=1, \ldots, 5)$ in such a way that $\sqrt{J_{2}(t)}$ can be estimated as:

$$
\sqrt{J_{2}(t, T)}=\sqrt{R_{1}^{2}+R_{2}^{2}+R_{3}^{2}+R_{4}^{2}+R_{5}^{2}}
$$




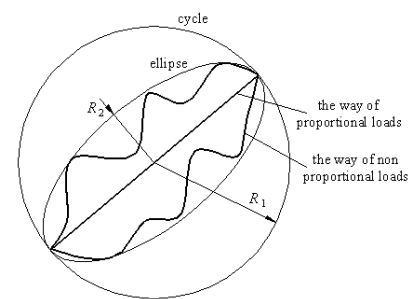

Figure 3 - Illustration of the ellipse and the cycle representing the path of $\sqrt{J_{2}(t)}$.

In order to evaluate the expression (22) the first step is to estimate the principal axes of $\xi_{5}$ representing the path of vector $\|s(t, T)\|$. This can be done by computing the variance matrix $\tilde{\boldsymbol{C}}_{s}$ representing the projection of vector $\|\boldsymbol{s}(t, T)\|$ on the principal axes of $\xi_{5}$ as follows:

$$
\tilde{\boldsymbol{C}}_{s}=\boldsymbol{A}_{s} \boldsymbol{C}_{\boldsymbol{s}} \boldsymbol{A}_{s}^{T}=\operatorname{diag}\left(\sigma_{1}^{2}, \ldots, \sigma_{5}^{2}\right)
$$

where $\boldsymbol{C}_{s}=E\left\{[\boldsymbol{s}(t, T)-\overline{\boldsymbol{s}}][\boldsymbol{s}(t, T)-\overline{\boldsymbol{s}}]^{T}\right\}$ and $\boldsymbol{A}_{s}=\left[\begin{array}{lllll}a_{1} & a_{2} & a_{3} & a_{4} & a_{5}\end{array}\right]$ represent, respectively, the covariance matrix and the orthonormal basis of eigenvectors associated to the stationary Gaussian random process $s(t, T)$, and $\sigma_{i}^{2}(i=1, \ldots 5)$ and $a_{i}(i=1, \ldots .5)$ are the variances and the directions for which the components of vector $s(t, T)$ are uncorrelated. Thus, the new components of vector $s(t, T)$ can be obtained as follows:

$$
\tilde{\boldsymbol{s}}(t, T)=\boldsymbol{A}_{s} s(t, T)
$$

where $\tilde{s}(t, T)=\left[\tilde{s}_{1}(t, T) \tilde{s}_{2}(t, T) \tilde{s}_{3}(t, T) \tilde{s}_{4}(t, T) \tilde{s}_{5}(t, T)\right]^{T}$.

The second step is the computation of the values of $R_{i}(i=1, \ldots, 5)$. First, it is necessary to estimate the variances of the projections of the time rates $\|\dot{\boldsymbol{s}}(t)\|$ and $\|\ddot{\boldsymbol{s}}(t)\|$ on the principal axes of the ellipsoid $\xi_{5}$ by using, respectively, the relations $\tilde{\boldsymbol{C}}_{\dot{s}}=\boldsymbol{A}_{s} \boldsymbol{C}_{\dot{s}} \boldsymbol{A}_{s}^{T}=\operatorname{diag}\left(\dot{\sigma}_{1}^{2}, \ldots, \dot{\sigma}_{5}^{2}\right)$ and $\tilde{\boldsymbol{C}}_{\ddot{s}}=\boldsymbol{A}_{s} \boldsymbol{C}_{\ddot{s}} \boldsymbol{A}_{s}^{T}=\operatorname{diag}\left(\ddot{\sigma}_{1}^{2}, \ldots, \ddot{\sigma}_{5}^{2}\right)$. Next, it is assumed that the components $\tilde{s}_{i}(t)$ are uncorrelated stationary Gaussian random processes in such a way that the values of $R_{i}=\max \left[\tilde{s}_{i}(t)-\overline{\widetilde{s}}_{i}\right]$ and their variances can be estimated as follows:

$$
\begin{gathered}
E\left[R_{i}\right] \approx \sigma_{i} \times\left(\sqrt{2 \ln N_{p}\left(\tilde{s}_{i}(t)\right)}+\frac{\gamma}{\sqrt{2 \ln N_{p}\left(\tilde{s}_{i}(t)\right)}}\right) \text { for } i=1, \ldots, 5 \\
V\left[R_{i}\right] \approx \sigma_{i} \times \frac{\pi^{2}}{6} \frac{1}{2 \ln N_{p}\left(\tilde{s}_{i}(t)\right)} \text { for } i=1, \ldots, 5
\end{gathered}
$$

where $N_{p}\left(\tilde{s}_{i}(t)\right)=\frac{T_{p}}{2 \pi} \sqrt{\frac{\ddot{\sigma}_{i}}{\sigma_{i}}}$ is the number of maxima associated to the components $\tilde{s}_{i}(t)-\overline{\widetilde{s}}_{i}$ for a period of time $T_{p}$, and $\gamma=0.5772$ is known as the Euler constant. Thus, the relation (22) enables to estimate the high cycle fatigue damage for a viscoelastic system subjected to Gaussian random loads, in which the term $\sqrt{J_{2}(t, T)}$ is estimated as:

$$
E\left[\sqrt{J_{2}(t, T)}\right] \approx \sqrt{E\left[R_{1}\right]^{2}+E\left[R_{2}\right]^{2}+E\left[R_{3}\right]^{2}+E\left[R_{4}\right]^{2}+E\left[R_{5}\right]^{2}}
$$

\section{NUMERICAL APPLICATIONS TO A PLATE TREATED WITH PASSIVE CONSTRAINED VISCOELASTIC LAYER}

To illustrate the main features of the proposed methodology of fatigue damage analysis as applied to engineering structures incorporating viscoelastic materials, numerical tests were performed using the FE model of a clampedclamped plate made of aluminum treated with a layer of 3M ISD112 ${ }^{\mathrm{TM}}$ viscoelastic material constrained between the base-plate and an outer aluminum sheet, as shown in Fig. 4. It is considered that the plate is subjected to concentrated transverse random load defined by a uniform power spectral density $\Phi_{f}(\omega)=1.1 \times 10^{6}(\mathrm{~Pa})^{2} / \mathrm{Hz}$ (white noise) applied 
at point $I$ indicated in the same figure. The FE model without viscoelastic treatment is composed of 140 elements and each viscoelastic treatment comprises the three-layer sandwich plate element developed according to Section 2 . The geometric dimensions in $x$ and $y$ directions are depicted in Fig. 4, and the thicknesses of the base-plate, the constraining layer, and viscoelastic core are, respectively, $1.0 \mathrm{~mm}, 0.5 \mathrm{~mm}$ and $0.0254 \mathrm{~mm}$. The material properties for the base-plate and the constraining layer are: Young modulus $E=70 \times 10^{9} \mathrm{~N} / \mathrm{m}^{2}$; mass density $\rho=2700 \mathrm{Kg} / \mathrm{m}^{3}$; Poisson's ratio, $v=0.3$. For the $3 \mathrm{M}^{\mathrm{TM}} \mathrm{ISD} 112\left(\rho=950 \mathrm{Kg} / \mathrm{m}^{3}\right)$ viscoelastic core the modulus function as shown in Fig. 2 is used.

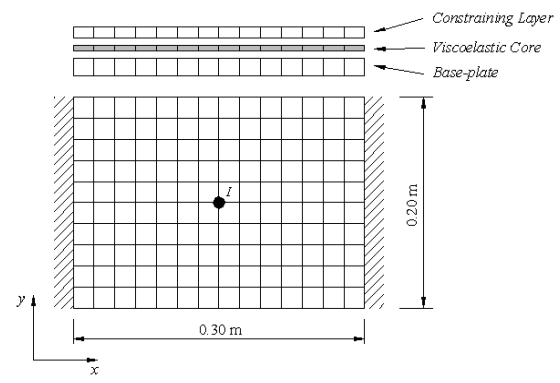

Figure 4- Illustration of the FE model of a supported plate with full surface treatment.

In this first application, the interest is to represent the distribution of the equivalent shear stress mode field $\sqrt{J_{2, a}}$ for each finite element (mean of the four nodal stress values) of the system without and with viscoelastic treatment for a period of time of $T_{p}=25000 \mathrm{~s}$. The values of the material mechanical properties used to perform the fatigue analysis are (Lambert et al., 2010): $t_{-1}=92 \mathrm{MPa}, f_{-1}=132 \mathrm{MPa}$ and $R_{m}=343 \mathrm{MPa}$. In order to compute the PSD matrix defined by Eq. (18), the reduced FRF $\hat{\boldsymbol{H}}_{I I}(\omega, T)$ has been computed assuming a temperature of the viscoelastic material of $25^{\circ} \mathrm{C}$, and a nominal basis $\boldsymbol{T}$ composed by 60 eigenvectors, one residual vector formed by the static displacements due to external forces and 54 residual vectors associated to the viscoelastic damping forces. Also, the reduced stress matrix for the base-plate is determined by considering the following isotropic material properties matrix:

$$
\boldsymbol{C}^{(1)}=\frac{E^{(1)} h^{(1)}}{1-v^{(1)^{2}}}\left[\begin{array}{ccc}
1 & v^{(1)} & 0 \\
v^{(1)} & 1 & 0 \\
0 & 0 & \frac{1-v^{(1)^{2}}}{2}
\end{array}\right]
$$

The fatigue behavior of the base-plate has been assumed deterministic with an equivalent endurance limit $f_{e q}=t_{-1}$ for zero mean loads (resulting in a zero mean hydrostatic pressure). Figures 5(a) and 5(b) show, respectively, the distributions of the equivalent shear stress mode for the plate without and with surface viscoelastic treatment. It can be noted that the dark areas represent the critical values of the mode which are associated with elements located in the central part of the plate due to the symmetric nature of applied force and the boundary conditions. Also, the most immediate consequence of the viscoelastic treatment is a reduction in the values of the equivalent shear stress mode enabling to evaluate the reliability of the structure from the Sines' criterion (21).

(a)

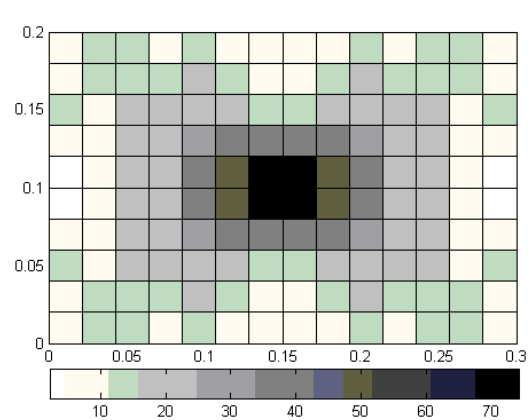

(b)

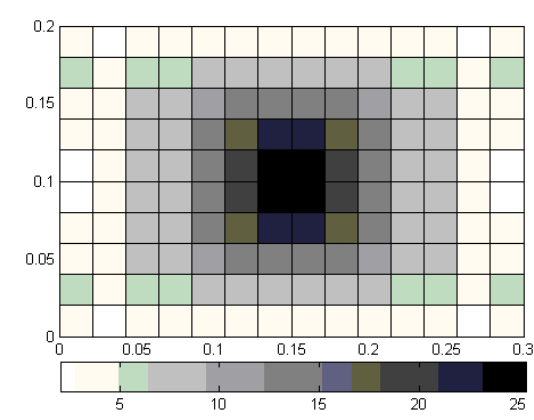

Figure 5-Mode of $\sqrt{J_{2, a}}$ : (a) without treatment: $74 M P a$; (b) with treatment: $25 \mathrm{MPa}$.

Figure 6 represent the PSD of the stress responses of the system without and with viscoelastic treatment, respectively, computed by taking into account the PSD of the load and the FRFs of the viscoelastically damped structure. 

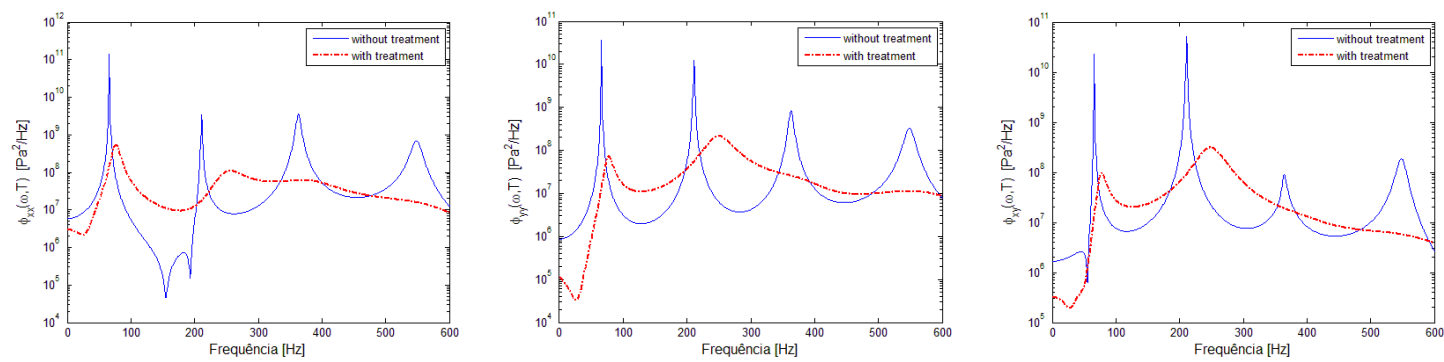

Figure 6 - PSD of the stress responses of the system without and with viscoelastic treatment.

To provide a sense of the efficiency of the viscoelastic treatment, Fig. 7 provides the comparison between the amplitudes of the FRFs (receptances) of the plate with and without viscoelastic treatment, enabling to evaluate the effectiveness of the viscoelastic patches for mitigating the displacement and stress response levels.

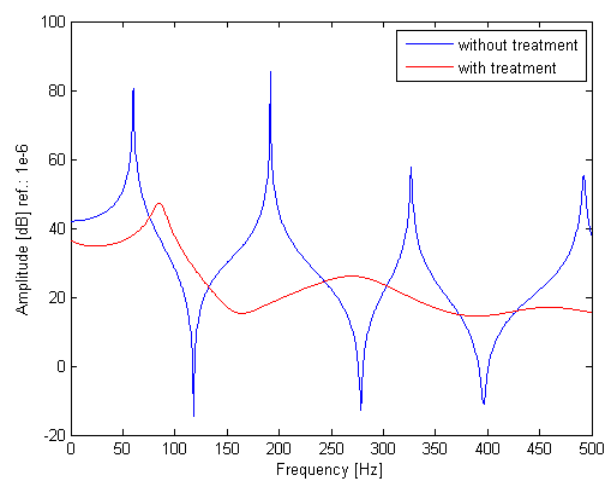

Figure 7 - FRF amplitudes of the plate without and with viscoelastic treatment.

In the case of random loads, it is convenient to take into account the uncertainties affecting the amplitude of the equivalent shear stress mode and the equivalent endurance limit on the probability approach of the Sines' criterion as defined in Eq. (21). In this context, a Gaussian distribution with a $10 \%$ coefficient of variation for the equivalent endurance limit $f_{e q}=t_{-1}=92 \mathrm{MPa}$ for has been considered for generating 500 Latin Hypercube samples (Florian, 1992). Moreover, in order to compute statistically the equivalent shear stress mode defined by Eq. (26), the random variables $R_{i}$ have been assumed to be uncorrelated with each other, having distributions described by their mode and variance according to the relations (25) and detailed in reference (Lambert et al., 2010).

Figure 8 shows the levels of the Sines' criterion for each finite element ranging from the minimum to the maximum estimated values obtained through simulations with the 500 samples. It can be perceived an augmentation in the estimated values of the reliability of the structure. These results enable to conclude about the interest in considering the random variability of the loads in the reliability analysis when compared with the deterministic one fixed at its mean value.

(a)

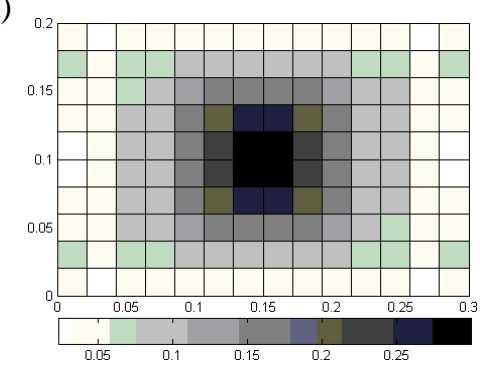

(b)

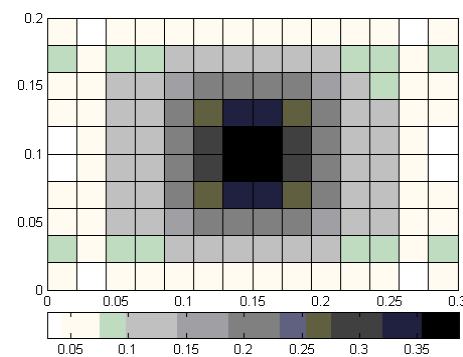

(c)

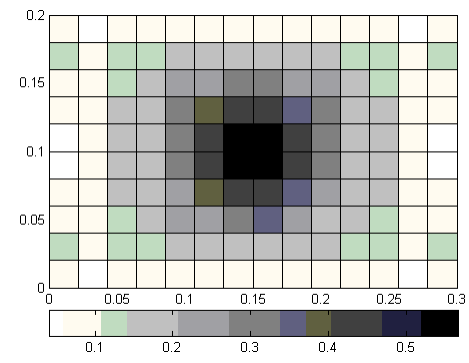

Figure 8 - Minimum (a), mean (b) and maximum (c) values of the estimations of the Sines' criterion for the viscoelastic system.

\section{CONCLUDING REMARKS}

A probability approach of the Sines' global criterion intended to be used for dealing with the fatigue damage analysis of viscoelastic structures subjected to Gaussian random loads was suggested and evaluated. The original aspects of the procedure lies in the adaptation of the concept of the probability approach of the stress-based global Sines' criterion, initially developed for undamped structures, for systems containing viscoelastic materials. 
The results obtained through the numerical simulations have demonstrated that the use of viscoelastic damping treatments increase the reliability of the structures by reducing their vibration levels. Also, from the numerical results one can conclude that the resulting criterion seems to be the most suitable for dealing with more realistic situations taking into account random loads to assess the reliability of the engineering structures.

Finally, an academic example from the aeronautic engineering interest was used to illustrate the proposed methodology enabling to estimate the distribution of the Sines' global indicator of such viscoelastic structure for a Gaussian process with the possibility to consider uncertainties on material parameters in which the reliability has been assessed in the critical areas through the spatial distribution of the expected equivalent shear stress amplitude.

\section{REFERENCES}

Bin Li and de Freitas, M., 2002, "A procedure for fast evaluation of high-cycle fatigue under multiaxial random loading," Journal of Mechanical Design, 124(3): 558-563.

Christensen, R.M., 1982, “Theory of viscoelasticity: An introduction.," Academic Press, Inc., New York.

Dang Van, 1993, "Macro-micro approach in high-cycle multiaxial fatigue," In: McDowell DL, Ellis R, editors. Advances in multiaxial fatigue. West Conshohocken: American Society for Testing and Materials, ASTM STP 1191: $120-30$.

de Lima, A.M.G., da Silva, A.R., Rade, D.A. and Bouhaddi, N., 2010, “Component mode synthesis combining robust enriched Ritz approach for viscoelastically damped structures," Engineering Structures, 32; 1479-1488.

Florian, A., 1992, "An efficient sampling scheme: updates Latin Hypercube sampling," Probabilistic Engineering Mechanics, 7(2):123-130.

Khatua, T.P. and Cheung, Y.K., 1973, "Bending and Vibration of Multilayer Sandwich Beams and Plates", International Journal for Numerical Methods in Engineering, 6:11-24.

Lambert, S., Pagnacco and Khalij, L., 2010, "A probabilistic model for the fatigue reliability of structures under random loadings with phase shift effects," International journal of fatigue, 32: 463-474.

Nashif, A.D. and Jones, D.I.G., 1985, "Vibration damping," John Wiley \& Sons, New York.

Palmeri, A. and Ricciardelli, F., 2006, "Fatigue analyses of buildings with viscoelastic dampers," Journal of Wind Engineering, 94:377-395.

Pitoiset, X. and Preumont, A., 2000, "Spectral methods for multiaxial random fatigue analysis of metallic structures," International Journal of Fatigue, 22: 541-50.

Rao, M.D., 2001, "Recent applications of viscoelastic damping for noise control in automobiles and commercial airplanes, "Proceedings of the Emerging Trends in Vibration and Noise Engineering Symposium, Columbus, USA.

Samali, B. and Kwok, K.C.S.,1995, "Use of viscoelastic dampers in reducing wind and earthquake induced motion of building structures," Engineering Structures, 17(9):639-654.

Sines, G.,1959, "Behaviour of metals under complex stresses," Sines G. \& Waisman J.L. editors.Metal fatigue, New York, McGraw-Hill, 145-69.

You, B.R. and Lee, S.B., 1996, “A critical review on multiaxial fatigue assessments of metals,” Int Journal Fatigue, 8(4): 235-44.

Weber, B., 1999, "Fatigue multiaxiale des structures industrielles sous chargement quelconque,” PhDThesis of INSA, Lyon.

\section{RESPONSIBILITY NOTICE}

The authors are the only responsible for the printed material included in this paper. 